\title{
Revisión Bibliográfica Actualizada sobre el Servicio Hotelero (2018)
}

\section{Updated Bibliographic Review About the Hotel Service (2018)}

*Guillermo Remigio Angamarca Izquierdo ${ }^{*}$ **Yelenis Díaz González², ***Carlos Cristóbal Martínez Martínez ${ }^{3}$

\section{${ }^{1}$ Universidad Técnica Estatal de Quevedo. Facultad de Ciencias Empresariales, Carrera de Marketing, Ecuador., 'Universidad Central "Marta Abreu” de Las Villas, ${ }^{3}$ Universidad Central "Marta Abreu” de Las Villas, Carretera a Camajuaní km 51ㄴ2. Santa Clara. Villa Clara. Cuba.}

*gangamarca@uteq.edu.ec,** yelenysdg@uclv.edu.cu,***cristobalc@uclv.edu.cu

Fecha de recepción: 28/01/2019 Fecha de aceptación: 26/03/2019 Publicado: 30/06/2019

\section{Resumen}

Este artículo aborda una revisión bibliográfica actualizada sobre las principales concepciones teóricas del servicio hotelero, que alcanza hasta el año 2018. Se han incluido publicaciones físicas y digitales, provenientes de libros, tesis y artículos científicos, relativos a él. Se propuso como objetivos específicos conceptualizar: 1. El servicio hotelero, 2. Características de los servicios hoteleros y 3. Elementos de la servucción hotelera. Se arribó a las siguientes conclusiones: servicio hotelero es un conjunto de actividades destinadas a brindar alojamiento temporal, a través de la interrelación entre el cliente y los empleados de servicio, los recursos físicos o productos y los sistemas de brindar servicios, según procesos, procedimientos y comportamientos, que generan servicios adicionales como alimentación, bebidas y entretenimiento, comunicación, entre otros, cuya finalidad es proporcionar satisfacción acorde a las necesidades y deseos de los clientes; las características del servicio hotelero son: intangibilidad, inseparabilidad, heterogeneidad, perecibilidad, integralidad, estacionalidad, origen geográfico de los clientes, la pluralidad de canales de comunicación, filosofía 24/365 y consumo del servicio en el lugar de producción; los elementos de la servucción hotelera son: 
el sistema de organización interna, el soporte físico y el personal en contacto, el cliente, los demás clientes y el servicio.

Palabras Clave: Marketing turístico, calidad de servicio, servicio hotelero

\begin{abstract}
This article deals with an updated bibliographical revision on the main theoretical conceptions of the hotel service, that reaches until the year 2018. Physical and digital publications have been included, coming from books, theses and scientific articles, related to it. It was proposed as specific objectives to conceptualize: 1 . The hotel service, 2. Characteristics of the hotel services and 3. Elements of the hotel service. The following conclusions were reached: hotel service is that aimed at providing temporary accommodation, through the interrelation between the client and the service employees, the physical resources or products and the systems of providing services, according to processes, procedures and behaviors, that generate additional services such as food, beverages and entertainment, communication, among others, whose purpose is to provide satisfaction according to the needs and desires of customers: the characteristics of the hotel service are: intangibility, inseparability, heterogeneity, perishability, integrality, seasonality, geographic origin of the clients, the plurality of communication channels, philosophy $24 / 365$ and consumption of the service in the place of production; The elements of the hotel servucción are: the system of internal organization, the physical support and the personnel in contact, the client, the other clients and the service.
\end{abstract}

Key Words: Touristic marketing, quality service, hotel service

\title{
Introducción
}

El turismo es una de las industrias de mayor importancia y crecimiento mundial. Su actividad económica se reconoce actualmente como la de más alta generación de empleo a escala global. En su aspecto histórico, en los últimos cinco años, los volúmenes de la demanda del 
turismo global han crecido notablemente. Según la Organización Mundial del Turismo (OMT), en el año 2018, esta demanda fue de 1 billón 530689 millones de dólares, que representa un 7\% más que en el 2017.

Esta oferta turística se satisface con el apoyo directo del sector hotelero. Los hoteles son empresas generadoras de servicios, o de servucción. Una característica importante de la servucción es la inseparabilidad del productor y del consumidor, en un mismo momento económico de la actividad productiva hotelera, lo que comporta la interrelación productor (hotel) y consumidor (huésped).

Uno de los propósitos del accionar empresarial eficiente de los hoteles es la satisfacción del cliente, objetivo que precisa el conocimiento de las principales concepciones teóricas sobre el servicio hotelero. Desde esta perspectiva, en este artículo se abordan, como resultado de una revisión de las fuentes secundarias especializadas, actualizadas al año 2018, las principales concepciones teóricas sobre el servicio hotelero, sus características diferenciales y los elementos del sistema de servucción, andamiaje teórico elemental para entender y proporcionar un servicio hotelero de calidad.

\section{El servicio hotelero}

Previo a su fundamentalización en el entorno de la hotelería, deviene necesario conceptualizar primeramente el servicio, por un lado, y el hotel, por otro, como elementos singulares y separados.

\subsection{Servicio}

Una definición universalmente aceptada de servicio no se ha logrado aún (Kotler, 1997; Díaz y Pons, 2010). Una conceptualización primaria aflora ligeramente a partir de la década del 70. Pero es a partir de la década del 80 donde se hacen verdaderos aportes al concepto. La tabla 1.1 muestra las principales definiciones de servicio. 
Tabla 1.

Definiciones de servicio

\section{Referencia}

\section{Definición de servicio}

\begin{tabular}{cl}
\hline AMA (1960) & "Son actividades, beneficios o satisfacciones que son ofrecidos a la \\
& venta o realizadas en unión con la venta de productos". \\
\hline Hill (1967) & "Es el cambio que una unidad productiva origina en la condición de una \\
& persona o de un bien perteneciente a ésta". \\
\hline Regan (1963) & "Pueden corresponder a intangibles que generan directamente \\
& satisfacciones (seguros), a tangibles que generan directamente \\
& satisfacciones (en los transportes o el inmobiliario) o a intangibles que \\
& generan satisfacciones cuando son adquiridos con productos o con otros \\
& servicios \\
& "Los servicios comerciales son las transacciones de una empresa o de un \\
& empresario con el mercado donde el objeto de esta transacción es una \\
& transferencia de propiedad de un bien tangible". \\
\hline Judd (1964) & "Un producto es una cosa y un servicio es un acto. El primero es un \\
& objeto, un artículo, un instrumento o un material... mientras que el \\
& último es una acción, una realización o un esfuerzo". \\
\hline Rathmell (1974)
\end{tabular}

\begin{tabular}{|c|c|}
\hline $\begin{array}{l}\text { Juran y Bingham (1974) } \\
\text { y Colunga }(1995 \text { b) }\end{array}$ & "Es un trabajo realizado para otros". \\
\hline Berry (1989) & $\begin{array}{l}\text { "Es en primer lugar un proceso y a diferencia de los artículos, no es un } \\
\text { objeto, sino una realización". }\end{array}$ \\
\hline $\begin{array}{l}\text { Eiglierd y Langeard } \\
\qquad(1989)\end{array}$ & $\begin{array}{l}\text { "Es la resultante de la interacción entre elementos de base, el cliente, el } \\
\text { soporte físico y el personal en contacto y constituye el beneficio que } \\
\text { debe satisfacer al cliente". }\end{array}$ \\
\hline Grönroos (1990) & $\begin{array}{l}\text { "Una serie de actividades de naturaleza más o menos intangible que } \\
\text { normalmente, aunque no necesariamente, se desarrolla mediante } \\
\text { interacciones entre el cliente y los empleados de servicios y/o los } \\
\text { recursos físicos o productos y/o sistemas de dar servicios que son } \\
\text { generados como soluciones a los problemas de los clientes". }\end{array}$ \\
\hline Flipo (1991) & $\begin{array}{l}\text { "Es el resultado de un acto o sucesión de actos de duración y } \\
\text { localización definidas, conseguido gracias a medios humanos y } \\
\text { materiales puestos a disposición de un cliente individual o colectivo, } \\
\text { según procesos, procedimientos y comportamientos codificados". }\end{array}$ \\
\hline Horovitz (1994) & $\begin{array}{l}\text { "Es el conjunto de prestaciones que el cliente espera como consecuencia } \\
\text { del precio, la imagen y la reputación del mismo". }\end{array}$ \\
\hline Lambin (1994) & $\begin{array}{l}\text { "Es todo acto o función que una parte puede ofrecer a otra, } \\
\text { esencialmente intangible, que no produce como resultado ninguna } \\
\text { propiedad, la cual puede o no vincularse a un producto físico". }\end{array}$ \\
\hline $\begin{array}{c}\text { Fisher y } \\
\text { Navarro (1994) }\end{array}$ & "Es el conjunto de actividades, beneficios o satisfactores que se ofrecen \\
\hline Payne (1996) & 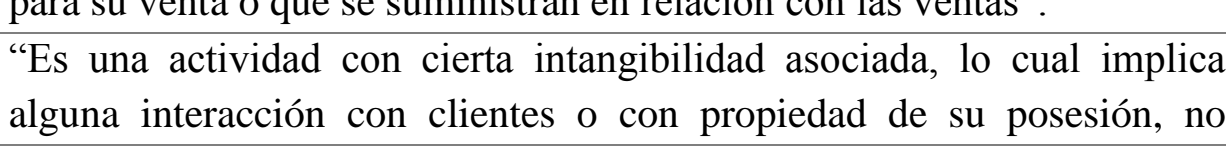 \\
\hline
\end{tabular}


resulta en una transferencia de propiedad. La producción de un servicio puede estar o no íntimamente asociado con un producto físico".

\begin{tabular}{|c|c|}
\hline Kotler (1997) & $\begin{array}{l}\text { "Es cualquier actividad o beneficio que una parte puede ofrecer a la otra, } \\
\text { esencialmente intangible, sin transmisión de propiedad. Su prestación } \\
\text { puede ir o no ligada a productos físicos". }\end{array}$ \\
\hline Peel (1999) & $\begin{array}{l}\text { "Es aquella actividad que relaciona la empresa con el cliente, la } \\
\text { finalidad es que éste quede satisfecho con dicha actividad". }\end{array}$ \\
\hline Gualier (2001) & $\begin{array}{l}\text { "Son todas aquellas actividades identificables, intangibles, que son el } \\
\text { objeto principal de una operación que se concibe para proporcionar la } \\
\text { satisfacción de necesidades de los consumidores". }\end{array}$ \\
\hline Cantú (2001) & $\begin{array}{l}\text { "Es una actividad o conjunto de actividades de naturaleza casi siempre } \\
\text { intangible, que se realizan mediante la interacción entre el cliente y el } \\
\text { empleado y/o instalaciones físicas de servicio, con el objeto de satisfacer } \\
\text { un deseo o necesidad". }\end{array}$ \\
\hline $\begin{array}{l}\text { Zeithmal y Bitner } \\
\qquad(2002)\end{array}$ & $\begin{array}{l}\text { "Es la inclusión de todas las actividades económicas cuyo resultado no } \\
\text { es producto de una construcción física, generalmente se consume en el } \\
\text { momento que se produce y proporciona valor agregado al añadir } \\
\text { aspectos (como la convivencia, entretenimiento, oportunidad, } \\
\text { comodidad o salud) que esencialmente son preocupaciones intangibles } \\
\text { para quien los adquiere por primera vez". }\end{array}$ \\
\hline $\begin{array}{l}\text { Stanton, Etzel y Walker } \\
\text { (2004) }\end{array}$ & $\begin{array}{l}\text { "Actividades identificables e intangibles que son el objeto principal de } \\
\text { una transacción ideada para brindar a los clientes satisfacción de deseos } \\
\text { o necesidades". }\end{array}$ \\
\hline Duque (2005) & $\begin{array}{l}\text { "Es el trabajo, la actividad y/o los beneficios que producen satisfacción a } \\
\text { un consumidor". }\end{array}$ \\
\hline Thompson (2006) & $\begin{array}{l}\text { "Son actividades identificables, intangibles y perecederas que son el } \\
\text { resultado de esfuerzos humanos o mecánicos que producen un hecho, un } \\
\text { desempeño o un esfuerzo que implican generalmente la participación del } \\
\text { cliente y que no es posible poseer físicamente, ni transportarlos o } \\
\text { almacenarlos, pero que pueden ser ofrecidos en renta o a la venta; por } \\
\text { tanto, pueden ser el objeto principal de una transacción ideada para } \\
\text { satisfacer las necesidades o deseos de los clientes". }\end{array}$ \\
\hline Bon (2008) & $\begin{array}{l}\text { "Es un medio para entregar valor a los clientes, facilitando los resultados } \\
\text { que los clientes quieren conseguir sin asumir costes o riesgos } \\
\text { específicos". }\end{array}$ \\
\hline
\end{tabular}

Díaz y Pons (2010) "Es una actividad de naturaleza más o menos intangible que normalmente, aunque no necesariamente, se desarrolla mediante interacciones entre el cliente y los empleados de servicios y/o los recursos físicos o productos y/o sistemas de dar servicios según procesos, procedimientos, comportamientos, que son generados para proporcionar satisfacción a los problemas y necesidades de los clientes".

Lovelock y Wirtz "Actividades económicas que crean valor y proporcionan beneficios a (2011) los clientes en tiempos y lugares específicos como resultado de producir 


\begin{tabular}{cl}
\hline & un cambio deseado en (o a favor) del receptor del servicio". \\
\hline Sandhusen (2012) & "Son actividades, beneficios o satisfacciones que se ofrecen en renta o a \\
& $\begin{array}{c}\text { la venta, y que son esencialmente intangibles y no dan como resultado la } \\
\text { propiedad de algo". }\end{array}$ \\
\hline Corea y Gómez (2014) & "Es una actividad o una serie de actividades de naturaleza más o menos \\
& intangible, que por regla general, aunque no necesariamente, se generan \\
& en la interacción que se produce entre el cliente y los empleados de \\
& servicios y/o los recursos o bines físicos y/o los sistemas de proveedores \\
& de servicios que se proporcionan como soluciones a los problemas del \\
& cliente". \\
"Salida de una organización con al menos una actividad necesariamente \\
llevada a cabo entre la organización y el cliente".
\end{tabular}

Fuente: Elaboración propia a partir de las definiciones de los autores.

Del análisis de las definiciones de servicio se puede llegar a la conclusión de que, por lo general, los autores abordan el servicio como un producto intangible y, otros, no obstante, lo relacionan directamente con bienes tangibles. Por otro lado, Eiglier y Langeard (1989); Grönroos (1990); Peel (1999); Gualier (2001); Cantú (2001); Stanton, Etzel y Walker (2004); Duque (2005); Thompson (2006); Bon (2008); Díaz y Pons (2010); Lovelock y Wirtz (2011); Corea y Gómez (2014) y Ballón (2016) abordan sobre todo el fin para el cual se realiza el servicio: la satisfacción de las necesidades de los clientes, la resolución de sus problemas y la creación de valor. A su vez, los conceptos aportados por Eiglier y Langeard (1989), Grönroos (1990), Flipo (1991), Cantú (2001), Díaz y Pons (2010), Corea y Gómez (2014) e ISO 9001:2005 (2015) hacen hincapié en los elementos para la prestación de un servicio. De otra parte, los conceptos señalados por Lambin (1994), Payne (1996), Kotler (1997), Thompson (2006), Sandhusen (2012) y Ballón (2016) hacen referencia a que los servicios no son objeto de transferencia de propiedad. Paralelamente, las definiciones realizadas por Berry (1989), Flipo (1991) y Díaz y Pons (2010) especifican cómo puede ser ofrecido el servicio: mediante procesos y procedimientos. Todos los aspectos citados con anterioridad deben conformar una 
definición acertada de servicio que hoy está ausente en la literatura investigada. Por tanto, el autor de esta investigación establece la siguiente definición de servicio:

Es una actividad esencialmente intangible cuyo resultado no es objeto de transferencia de propiedad, se desarrolla mediante interacciones entre el cliente y los empleados de servicios y/o los recursos físicos o productos y/o sistemas de brindar servicios, según procesos, procedimientos y comportamientos, cuya finalidad es proporcionar satisfacción acorde a las necesidades y deseos de los clientes.

\subsection{Hotel}

Los hoteles son la base tangible sobre la que se asienta la industria turística. Por consiguiente, resulta conveniente estudiar los conceptos relacionados con este término. La tabla 1.2 evidencia las principales definiciones de hotel.

Tabla 2.

Definiciones de hotel

\begin{tabular}{|c|c|}
\hline Referencia & Definición de hotel \\
\hline $\begin{array}{l}\text { Musa y Sanchis } \\
\text { (1993) y Cortina y } \\
\text { otros (2002) }\end{array}$ & $\begin{array}{l}\text { "Establecimiento que facilita alojamiento con o sin servicios } \\
\text { complementarios, distinto de los correspondientes a cualquiera de otras } \\
\text { modalidades". }\end{array}$ \\
\hline Muñc & $\begin{array}{l}\text { "Establecimiento que facilita el alojamiento con o } \text { sin servicios } \\
\text { complementarios". }\end{array}$ \\
\hline $\begin{array}{l}\text { Figuerola y otros } \\
\text { (1996) y Figuerola } \\
\text { (1997) }\end{array}$ & $\begin{array}{l}\text { "Establecimiento de alojamiento que cuenta con una mayor variedad de } \\
\text { servicios, fluctuando el mismo según su categoría". }\end{array}$ \\
\hline Mince & $\begin{array}{l}\text { "Es un edificio planificado y acondicionado para albergar personas } \\
\text { temporalmente y que permite a los viajeros alojarse durante sus } \\
\text { desplazamientos". }\end{array}$ \\
\hline in (2004) & $\begin{array}{l}\text { "Lugar que proporciona alimentación y hospedaje, un lugar de } \\
\text { entretenimiento para el viajero y que opera bajo una fase para obtener } \\
\text { utilidades". }\end{array}$ \\
\hline Blasco y & $\begin{array}{l}\text { "Empresa dedicada de manera profesional o habitual, a proporcionar } \\
\text { habitación a las personas con o sin servicios complementarios, a cambio de } \\
\text { un precio". }\end{array}$ \\
\hline
\end{tabular}


Navarro (2008) "Es un establecimiento dirigido por profesionales en el que se ofrece al cliente alojamiento con o sin servicios integrados, a cambio de una contraprestación económica".

Talaya y Lara (2008) "Establecimiento comercial abierto al público que, ofreciendo alojamiento y Paniagua (2014) turístico, con o sin comedor y otros servicios complementarios, ocupa la totalidad de un edificio o parte independizada del mismo

Lattin (2009) "Un edificio o una institución que proporciona alojamiento, comidas y servicios al público".

de la Torre (2009) "Es una institución de carácter público que ofrece al viajero alojamiento, alimentos y bebidas, así como entretenimiento, la cual opera con la finalidad de obtener utilidades".

\begin{tabular}{cl}
\hline Loida (2011) & "Es la industria que se ocupa de proporcionar a los clientes alojamiento, \\
& comida y otros servicios, mediante pago". \\
\hline Di Muro (2012) & "Un establecimiento de carácter público, destinado a dar una serie de \\
& servicios, alojamiento, alimentos, bebidas y entretenimiento". \\
\hline Sanisaca (2012) & "Es el conjunto de todos aquellos establecimientos comerciales que de \\
& forma profesional y habitual, prestan servicios de hospedaje y restauración, \\
& ya sean habitaciones o apartamentos, con o sin otros servicios \\
& complementarios". \\
& "Es un lugar que proporciona alimentación y hospedaje que ofrezca \\
& entretenimiento a los viajeros y que opera bajo la base de obtener \\
& utilidades". \\
\hline Daza (2013) & "Es un edificio planificado y acondicionado para otorgar servicios de \\
& alojamiento a las personas temporalmente y que permite a los huéspedes \\
& sus desplazamientos, además de ofrecerles servicios adicionales". \\
\hline "Es el establecimiento que presta el servicio de hospedaje en unidades \\
habitacionales amuebladas, cuenta con servicio de recepción, servicio \\
sanitario privado, servicios de alimentos y bebidas y otros servicios \\
adicionales". \\
"Es todo establecimiento que de modo habitual, mediante precio, preste al \\
público en general, servicios de alojamiento, comidas y bebidas y que \\
reúna, además las condiciones necesarias para la categoría que le \\
corresponde".
\end{tabular}

Fuente: Elaboración propia a partir de las definiciones de los autores.

Del análisis de la tabla anterior se concluye que todos los autores citados mencionan que un hotel es un establecimiento destinando a brindar alojamiento, hospedaje, habitación o albergue. Solo Mincetur (2004) señala el carácter temporal de ese alojamiento, lo cual es primordial para el concepto de hotel que se asuma en cualquier investigación. Por otro lado, los autores Musa y Sanchis (1993), Muñoz (1994), Figuerola y otros (1996), Figuerola 
(1997), Cortina y otros (2002), Blasco y otros (2006), Navarro (2008), Talaya y Lara (2008),

Lattin (2009), Loida (2011), Sanisaca (2012), Arias (2014), Paniagua (2014) y Hernández (2016) plantean además que los hoteles ofrecen servicios complementarios, adicionales, variados o integrales. Sin embargo, Barragán (2004), Lattin (2009), de la Torre (2009), Loida (2011), Di Muro (2012), Sanisaca (2012), Daza (2013), Hernández (2016) y Veloz y Vasco (2016) detallan esos servicios complementarios que se ofertan: específicamente alimentación, bebidas y entretenimiento. Este último, en la actualidad, se ha complementado con el uso de las Técnicas de la Información y la Comunicación (TIC). Por otra parte, en los conceptos anteriores se señala que la finalidad de un hotel es la obtención de utilidades, así lo plantean Barragán (2004), Blasco y otros (2006), Navarro (2008), de la Torre (2009), Loida (2011), Daza (2013) y Veloz y Vasco (2016), lo cual no está en correspondencia con el concepto de servicio asumido en la investigación.

Con base en las definiciones analizadas, integrando los conceptos de servicio y hotel, estos autores concluyen que:

Servicio hotelero es un conjunto de atributos que se proporciona en un hotel, destinado a brindar alojamiento temporal, a través de la interrelación entre el cliente y los empleados de servicios, los recursos físicos o productos y los sistemas de brindar servicios, según procesos, procedimientos y comportamientos, que generan servicios adicionales como alimentación, bebidas y entretenimiento, comunicación, entre otros, cuya finalidad es proporcionar satisfacción acorde a las necesidades y deseos de los clientes. (Barragán, 2004; Blasco et al., 2006)

\section{Características de los servicios hoteleros}

Se consideran características básicas de los servicios hoteleros: Intangibilidad (el servicio no puede ser percibido por los sentidos), inseparabilidad (los servicios se producen y consumen al mismo tiempo), heterogeneidad (los servicios son de difícil estandarización), perecibilidad 
(los servicios no se pueden almacenar). De la Varga y Galindo (2007), Kotler y otros (2011), Gomes de Souza y otros (2012), Mariño y otros (2013), Monsalve y Hernández (2015), Duque y Parra (2015), Román (2015) y Ballón (2016) coinciden en que las cuatro características mencionadas con anterioridad son básicas para el servicio hotelero. Sin embargo, Musa y Sanchis (1993), Aguayo (1997), Mestres (1999), Benitez (2010), De Cruz (2012), Bloggers (2013) y Llois (2016) agregan otra característica importante para el servicio hotelero, la integralidad, que implica que el producto hotelero se puede integrar con varios productos turísticos muy relacionadas unos con otros: avión, hotel, restauración, alquiler de vehículo, entre otros. De Cruz (2012) añade la característica de estacionalidad en el servicio hotelero que involucra la concentración de la demanda turística en ciertos períodos del año, es decir, están sujetos a ciclos de demanda muy alta y ciclos de demanda muy baja. Por otro lado, Musa y Sanchis (1993), Aguayo (1997) y Mestres (1999) adicionan como otras características del servicio hotelero: la diversidad del origen geográfico de los clientes, la pluralidad de canales de comunicación que pueden ser utilizados por éstos para la contratación de los servicios y la permanencia en las operaciones durante las 24 horas sin interrupción. Estos mismos autores señalan que para el caso del servicio hotelero, este no puede ser trasladado al cliente, debe ser éste quien se desplace al lugar de la prestación del servicio es decir, el servicio se consume en el lugar de producción.

Por lo arriba expuesto, es criterio del autor de este artículo que las características del servicio hotelero son: intangibilidad, inseparabilidad, heterogeneidad, perecibilidad, integralidad, estacionalidad, origen geográfico de los clientes, la pluralidad de canales de comunicación, filosofía 24/365 y consumo del servicio en el lugar de producción.

\section{Elementos de la servucción hotelera}

En los hoteles se da el proceso de servucción o la generación de los servicios hoteleros. Cuando existe un nivel de contacto elevado entre clientes y la empresa, como es el caso de los 
servicios hoteleros, el proceso de producción del servicio, o sea, su sistema de servucción ha de contar con seis elementos (figura 1.2) que son los que forman este sistema: cliente, soporte físico, personal en contacto, servicio, sistema de organización interna y demás clientes (Eiglier y Langeard, 1975; Bernal, 2006; Salinas, 2006 a y b; Mejía y otros, 2008; Rico y otros, 2008). Tres elementos pertenecen a la empresa de servicio hotelero: el sistema de organización interna, el soporte físico y el personal en contacto; dos elementos pertenecen al mercado: el cliente y los demás clientes y un elemento que es el resultado de la interacción de los clientes con el soporte físico y el personal en contacto: el servicio. Todo esto está relacionado con la teoría general de los servicios.

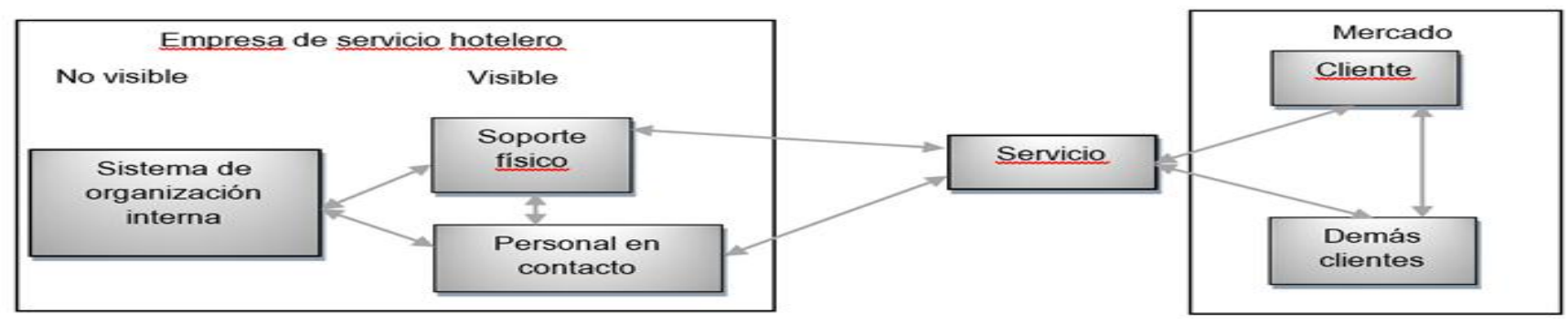

Figura 1. El proceso de producción de un servicio hotelero como sistema. Adaptado de Eiglier y Langeard (1993).

El servicio hotelero constituye el objetivo del sistema. Este es el resultado de la interacción entre los tres elementos base: el cliente, el soporte físico y el personal hotelero en contacto. Esta resultante constituye el beneficio que ha de satisfacer la necesidad del cliente y el ofrecimiento de un servicio de calidad. El cliente hotelero resulta ser un elemento primordial del sistema de servucción. Constituye el beneficiario del servicio y su presencia en el hotel es absolutamente indispensable pues, sin este, el servicio no puede existir. Por tanto, la empresa de servicio hotelero debe estudiar las necesidades, deseos, expectativas y el comportamiento del cliente para satisfacerlo.

Por su parte, el soporte físico y el personal en contacto directo con el cliente constituyen la parte visible del sistema para el cliente. Es el soporte material necesario para la producción de un servicio hotelero y del que se sirven el personal en contacto y/o los clientes. Este soporte 
físico puede escindirse en dos grandes categorías: los instrumentos necesarios para realizar el servicio hotelero y el entorno material en que éste se desarrolla. El personal en contacto es la persona o las personas empleadas por la empresa de servicio hotelero y cuyo trabajo requiere estar en contacto directo con el cliente. Este personal tiene un doble papel: una función operacional y una función relacional. La función operacional es el conjunto de operaciones hoteleras que deben ser efectuadas por este personal y que son traducidas por instrucciones muy precisas dadas a este personal. La función relacional es el desempeño de manera agradable de sus funciones. Tres conjuntos de elementos constituyen lo relacional: lo visible, lo gestual y lo verbal. Lo visible, es ante todo lo que el cliente ve, la limpieza, el orden y el buen estado del soporte físico a disposición del personal, también es todo lo que concierne a la apariencia del personal, el físico, la limpieza, el pelo, la forma de maquillarse. Lo gestual, está constituido por el comportamiento del personal en contacto, es estar inmediatamente disponible, tener unos gestos precisos y armoniosos para realizar las operaciones, que sonría en el momento oportuno, al menos al principio y al final de la interacción y que mire a los ojos del cliente cuando le habla. Lo verbal, lo constituyen las expresiones verbales que ha de utilizar el personal, las formas de educación y cortesía, las expresiones de bienvenida, concisión y precisión de las frases de naturaleza operacional; la nitidez del timbre, la exactitud y el profesionalismo del tono. Todos estos elementos revisten una gran importancia para el cliente, deben ser objeto de un control preciso y riguroso por parte de la dirección, además de invertir en un programa de formación que tome en cuenta el campo operacional y lo relacional. Por consiguiente, el personal en contacto debe contribuir a la satisfacción del cliente (Pérez, 2002; Botero, 2003-2005; Doucet, 2004; Quijano, 2004; Tsaur y Lin, 2004; Sharma y Mehta, 2004; Gayathri y otros, 2005; Ramírez y otros, 2005; Leal, 2006; Gallarza y Gil, 2006; Cordero, 2006; Serrano y otros, 2007; Saris y Gallhofer, 2007; Javaras y Ripley, 2007; Martínez y Martínez, 2008; Munguía y otros, 2008; Alvarado y otros, 2008). Para 
Crosby y Stephens (1987); Parasuraman y otros (1988); Eiglier y Langeard (1989); Bitner (1990); Grönroos, (1994); Hartline y Ferrell (1996); Kelley y Hoffman (1997); Necmi (1999); Bettencourt y otros (2001); Spencer-Matthews y Lawley (2006). El personal de contacto, constituye, sin duda, un elemento crítico para la realización de un servicio de calidad en el sector hotelero.

El sistema de organización interna, a su vez, está constituido por todas las funciones clásicas de la empresa: finanzas, marketing, operaciones, personal pero también, por algunas funciones específicas que son necesarias para la realización de un servicio hotelero de calidad. Este sistema constituye la parte no visible para el cliente y ha de estar bien coordinado pues va a tener una influencia directa sobre el soporte físico y sobre el personal en contacto, los cuales afectan la percepción de calidad y de satisfacción del cliente. Los demás clientes son los que al mismo tiempo se encuentran para recibir un servicio, porque es raro que un servicio sea ofrecido a un solo cliente. Se establecen relaciones entre estos porque están presentes en el mismo hotel y pueden influir en la calidad del servicio prestado al otro cliente.

En resumen, tal como se muestra en la figura 1.3, el conocimiento de la conceptualización de servicio hotelero, de las características diferenciales y la clasificación de este servicio y los elementos del sistema de servucción, constituyen aspectos básicos para proporcionar un servicio hotelero de calidad.

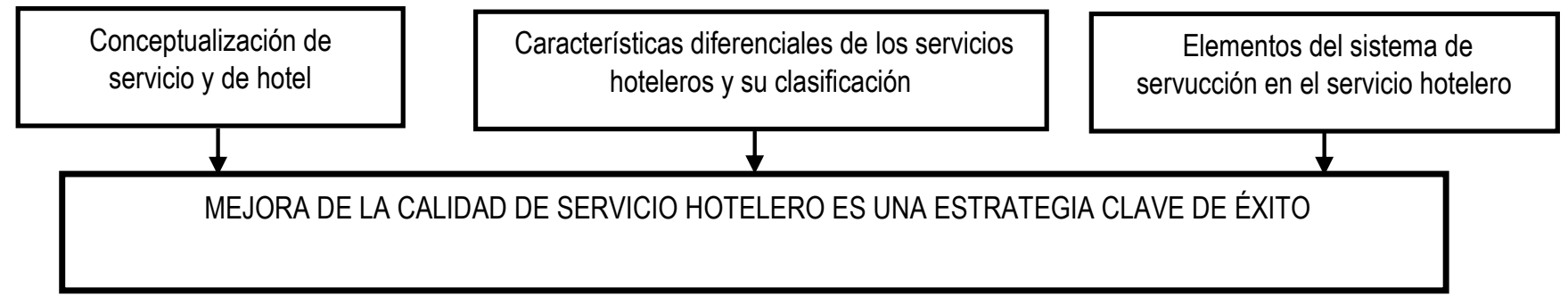

Figura 2. Elementos básicos para proporcionar servicio hotelero de calidad. Elaboración: los autores.

\section{Conclusiones}


Se arribó a las siguientes conclusiones: servicio hotelero es un conjunto de atributos destinado a brindar alojamiento temporal, a través de la interrelación entre el cliente y los empleados de servicios, los recursos físicos o productos y los sistemas de brindar servicios, según procesos, procedimientos y comportamientos, que generan servicios adicionales como alimentación, bebidas y entretenimiento, comunicación, entre otros, cuya finalidad es proporcionar satisfacción acorde a las necesidades y deseos de los clientes: las características del servicio hotelero son: intangibilidad, inseparabilidad, heterogeneidad, perecibilidad, integralidad, estacionalidad, origen geográfico de los clientes, la pluralidad de canales de comunicación, filosofía 24/365 y consumo del servicio en el lugar de producción; los elementos de la servucción hotelera son: el sistema de organización interna, el soporte físico y el personal en contacto, el cliente, los demás clientes y el servicio.

\section{Referencias bibliografícas}

Acosta, D. (2007). “Gerencia de producción del servicio”. Presentación. Curso de postgrado. ISPJAE. Cuba. 10-15 octubre.

Aguayo Moral, J. (1997): Un modelo para la segmentación de la información contable en las empresas hoteleras. Tesis doctoral. Universidad de las Palmas de Gran Canaria.

Alvarado, N. F.; Mayorga, X. J.; Mendoza, L. F. y Hernández, D. (2008). "Investigación sobre satisfacción del cliente de servicios de seguridad de Chinandega". Trabajo de Diplomado. Universidad de Managua.

Arias, A. (2011). El enfoque a los clientes. Calidad de los servicios: La filosofía de Parasuraman. Madrid: Facultad de Ciencias de la Documentación.

Ballón, S. C. (2016). Evaluación en la calidad de servicio y satisfacción del cliente en los hoteles de 2 y 3 estrellas de la cuidad de Abancay. Tesis presentada en opción al título de licenciada en administración y finanzas. Universidad ESAN, Lima, Perú. 
Barragán, L. (2004). Hotelería. México: Instituto Politécnico Nacional. http://itzamna.bnct.ipn.mx/dspace/bitstream/123456789/6887/1/MODELOCRM.pdf

Barrientos, C. (2017) Plan de mejora organizacional como factor determinante de la calidad de servicio del hotel Perú pacífico en Jesús María. repositorio.uwiener.edu.pe

Benítez, J. (2010). La calidad del servicio en la industria hotelera. Tesis doctoral en ciencias económicas y empresariales. Universidad de las palmas de gran canaria, España.Recuperado el 22/04/2013, de: http://acceda.ulpgc.es/bitstream/10553/ 5516/1/0628694_00000_0000.pdf

Bernal, J. (2006) Servucción: el proceso de creación de servicios. [En línea]. Disponible en: www.mercadeo.com [Accesado el 14 de marzo de 2008].

Bethencourt, M.; Díaz, F. M.; González, M. O. y Sánchez, J. (2005). “La medición de la calidad de los servicios prestados por los destinos turísticos: el caso de la isla de La Palma”. Revista de Turismo y Patrimonio Cultural. Volumen 3. Número 2.

Bitner, M. (1990). Evaluating service encounter: the effects of physical surroundings and employee responses. N.Y: Journal of Marketing 54, 69-82.

Blasco, A., Bachs, J., Bancells, J., \& Vives, R. (2006). Manual de gestión de produccion de alojamiento y restauracion. Madrid: Editorial Sintesis.

Bon (2008). Gestión de Servicios. Primera edición. Editorial del gobierno Británico. Reino Unido. p.p. 21

Botero (2006). "Calidad en el servicio: el cliente incógnito". Revista Suma Psicológica. Volumen 13. Número 2.

Cantú, H. (2006). Desarrollo de una cultura de calidad. (3ra. Ed.). México, D. F.: McGraw Hill.

Cordero, A. L. (2006). "Servicio y atención al cliente en la refresquería Candy". Trabajo de Diplomado. Universidad de Managua. Nicaragua. 
Corea, L. M. y Gómez, S. J. (2014). El posicionamiento y la segmentación del mercado de las empresas de servicios. Tesis presentada en opción al título de licenciada en mercadotecnia. Universidad Nacional Autónoma de Nicaragua.

Cortina, F.; Martínez, A. y Várela, B. (2002). Aproximación a la investigación del alojamiento privado de uso turístico. Estudios Turísticos, No. 151, pp. 87-97.

Daza J. (2013). Análisis de la Medición de Calidad en los Servicios Hoteleros. Criterio Libre. Año 11. No. 19. Bogotá (Colombia). Julio-Diciembre 2013. ISSN 1900-0642

De la Cruz, P. (2012). Contribución a los Modelos y Metodologías para la Estimación de la Calidad Percibida por los Usuarios (QoE) a partir de Parámetros de Calidad de Red/Servicio (QoS) en Servicios Convergentes Multimedia (Triple-Play). Tesis (Doctoral), E.T.S.I. Telecomunicación (UPM).

De la Torre, Francisco (2009), https://www.monografias.com/trabajos89/monografiahoteles/monografia-hoteles.shtml

De la Varga, J. M. y Galindo, F. C. (2007). La técnica del incidente crítico como método de validación de las dimensiones de la calidad en el sector hotelero. Empresa global y mercados locales: XXI Congreso Anual AEDEM, Universidad Rey Juan Carlos, Madrid, 6,7 y 8 de junio de 2007, pág. 15

Deming, E. (1990). Four-day Dr Deming Seminar, Jan 30-Feb 2 1990, Washington, D.C

Di Muro, L. (2012). Manual práctico de recepción hotelera. 2a ed. México, D. F.: Trillas. 224 p.: il.

Díaz Gonzáles, Y. y Pons García, R. (2008). (2008). El Marketing y la Calidad de Servicio Percibida: Marco Conceptual. Tesis doctoral. Santa Clara: UCLV

Díaz Gonzáles, Y. y Pons García, R. (2009). Conceptualización y modelización de la calidad de servicio percibida: análisis crítico. Villa Clara: Universidad Central "Marta Abreu" de Las Villas. 
Duque, E. (2005). Revisión del concepto de calidad del servicio y sus modelos de Medición. INNOVAR, Revista de Ciencias Administrativas Y Sociales, 15, 18. Retrieved from http://www.redalyc.org/pdf/818/81802505.pdf

Eiglier, P. \& Langeard, E. (1989). Servucción. El marketing de servicios. Madrid: McGraw-Hill.

Fernández, M. (2016). "Validación de Servqual como instrumento de la calidad de servicio bancario". Revista Europea de Dirección y Economía de la Empresa. Volumen 9. Número 1.

Fick, R. y Ritchie, R. (1991). Measuring service quality in the travel and tourism Industry. Journal of travel research. (Fall), 2-9.

Figuerola, M., 1997. Elementos para el estudio de la economía de la empresa turística. Editorial Síntesis, Madrid.

Figuerola, M., Arranz, A., Prado, J., Allende, E., 1996. Incidencia y efectos multiplicadores del turismo en la economía. Información Comercial Española 749, $17-31$.

Flipo, P. (1991). La gestión del marketing de servicios. Ediciones Granica. Argentina.

Gallarza, M. G. y Gil, I. (2006). "Value dimensions, perceived value, satisfaction and loyalty: an investigation of university student's travel behavior". Tourism Management. Número 27.

Gayathri, H.; Vinaya, M. C. y Lakshmisha, K. (2005). "A pilot study on the service quality of insurance companies". Journal of Services Research. Volumen 5. Número 2. Octubre.

Gomes de Souza, A.; De Azevedo, M. L.; Kovacs, M. H. y Queiroz, R. (2012). Percepción del riesgo en la elección de servicios hoteleros en el ámbito virtual: 
Actitud de los consumidores frente al uso de estrategias de reducción de riesgos. Estudios y perspectivas en turismo. v. 21, n. 1, p. 52-67.

Grönroos, C. (1984). A service quality model and its marketing implications. European Journal of Marketing, 18, 36-44.

Grönroos, C. (1990). Service management and marketing. Lexington, Mass.: Lexinggton Books.

Gualier, H. (2001). “Mercadeo en los servicios de información”. [En línea]. Disponible en: http://www.monografías.com [Accesado el 21 de Abril de 2005].

Hartline, M. y Ferrell, O. (1996). The management of customer-contact service employees: an empirical investigation. The Journal of Marketing, 1996 - JSTOR

Hernández, E. (2016). Proyección de la calidad de los servicios en el Sistema de Dirección y Gestión de la Empresa "Hotel Los Caneyes". Trabajo de diploma de la carrera de Ingeniería Industrial. CDICT. UCLV.

Ishikawa, K. (1988). ¿Qué es el control total de la calidad? La modalidad japonesa. Edición Revolucionaria. La Habana.

Javaras, K. N. y Ripley, B. D. (2007). “An unfolding latent variable model for likert attitude data: drawing inferences adjusted for response style". Journal of the American Statistical Association. Volumen 102. Número 478.

Kelley, S. W. y Turley, L. W. (2001). “Consumer perceptions of service quality attributes at sporting events". Journal of Business Research. Número 54.

Kotler, P., Bowen, J. T., Makens, J. C., Miranda, J. G. M. \& Zamora, J. F. (2011). Marketing turístico. (5ta ed.). Madrid: Pearson Education.

Kotler, Ph. (1997). Marketing management: analysis, planning and control. 9th edition. Editorial Prentice- Hall International. USA.

Lambin, J. (1994). Marketing estratégico. Editorial McGraw-Hill. Madrid. 
Lattin. (2007). La industria del hospedaje y el servicio de alimentos. Columbia: Planeta.

Leal, J. A. (2006), “Buscando la satisfacción y lealtad de los clientes en colaboración de nuestro cliente interno". Disponible en: http://www.gestiopolis.com [Accesado el 10 de febrero de 2007].

Llois, A. (2016). Características del Producto Hotelero. https://forotourism.wordpress.com/2016/01/07/caracteristicas-del-producto-hotelero/

Lockwood, A. (1994) Using Service Incidents to Identify quality improvement points . N.Y. MCB UP Ltd.

$\begin{array}{lllll}\text { Loida, } & \text { E. } & \text { (2011). } & \text { Turismo } & \text { y }\end{array}$ https://www.monografias.com/trabajos89/monografia-hoteles/monografiahoteles.shtml

Lovelock, C. y Wirtz, J. (2011). Marketing de Servicios. (7ma ed.) México, D.F.: Pearson.

Mariño, J. A.; Sánchez, M. J.; Martín, F. A. y Hernández, J. M. (2013). Calidad en el sector turístico. Análisis del estado del arte. En Turismo e innovación: VI jornadas de investigación en turismo (291-312), Sevilla: Facultad de Turismo y Finanzas. https://idus.us.es/xmlui/bitstream/handle/11441/52953/marino-romero.pdf

Martínez, J. A. y Martínez, L. (2008). “La medición de la calidad percibida en servicios deportivos; un enfoque en primera persona". Revista Internacional de Medicina y Ciencias de la Actividad Física y el Deporte. Volumen 8. Número 31.

Mejía, S. M.; Ruiz, R. E.; Guillén, U. y Wilson, R. A. (2013 "Investigación sobre el proceso de servicio en talleres Casa Pellas de Managua”. Trabajo de Diplomado. Universidad de Managua.

Mestres Soler, J. (1999): Técnicas de Gestión y Dirección Hotelera. $2^{\mathrm{a}}$ ed., Barcelona: Ediciones Gestion 2000. 
Mincetur (2004). Reglamento de establecimientos hoteleros. https://es.slideshare.net/oscarlopezregalado/establecimientos-hoteleros

Monsalve, C. y Hernández, S. I. (2015). Gestión de la calidad del servicio en la hotelería como elemento clave en el desarrollo de destinos turísticos sostenibles: caso Bucaramanga. Revista Escuela Administración de Negocios. No. 78, January/June. Bogotá.

Munguía, A. B.; Betanco, M. M.; Duarte, C. H.; Gómez, L. O. y Orozco, C. M. (2008). “Investigación sobre satisfacción del cliente en la Sala de Bellezas Mercedes". Trabajo de Diplomado. Universidad de Managua.

Muñoz Oñate, F., 1994. Marketing Turístico. Editorial Centro de Estudios Ramón Areces, Madrid.

Musa Alhasan, S.M. y Sanchis Palacio, J.R. (1993): "La determinación de los precios de alojamiento en la industria hotelera" Estudios Turísticos. Instituto de Estudios Turísticos D.G. de Política Turística, n¹17, pp. 49 a 60. Madrid.

Navarro (2008). Gestión de hoteles, hostelería y turismo. Editorial vértice. España.

Necmi, K. (1999); Quality customer service demands human contact. International Journal of Bank Marketing. Emeraldinsight.com

Paniagua, J. A. (2014). Concepto y tipos de hotel. https://es.slideshare.net/tonypaniagua94/conceptos-y-tipos-de-hotel

Parasuraman, A., Zeithaml, V. A., Berry, L. (1988). SERVQUAL a multiple-item scale for measuring consumer perceptions of service quality. N.Y: Journal of Retailing 64(1), 12-40.

Payne, A. (1996). La esencia de la mercadotecnia de servicios. Editorial Prentice-Hall Iberoamericana. México. 
Peel, M. (1999). Cultura de servicios y calidad percibida. Mercadeo y ventas. Ediciones Deusto, S.A. Bilbao.

Pei Mey, L., Akbar, K., y Yong Gun Fie, D. (2005). Service quality: a study of the luxury hotels in Malaysia. Journal of American Academy of Business, 7(2), 46-55

Pérez, J. y Parra, C. (2007). "Evaluación y análisis de la calidad del servicio de apoyo desde la perspectiva del usuario: primer paso hacia la confiabilidad". Ind. Data. Volumen 10. Número 1.

Ramírez, E.; Rodríguez, A. y Zapata, E. (2005). "Influencia de las diferencias individuales en la calidad de los servicios universitarios". Revista Colombiana de Marketing. Volumen 4. Número 6.

Ravichandran, M., Tamil, S., Kumar, S. Prabhakaran, S. Influence of service quality on customer satisfaction application of SERVQUAL model. International Journal of Business \& Management, 5 (4) (2010), pp. 117-124

Rico, B. J.; Castillo, I. A.; Meneses, X. C. y Sánchez, K. M. (tutora) (2008). “Investigación sobre la calidad de servicio en el proceso de atención al cliente en la librería San Jerónimo, sucursal Ciudad Jardín”. Trabajo de Diplomado. Universidad de Managua.

Román, R. (2015). Los Servicios Personalizados en la Hotelería.

Salinas, O. (2006a). "El problema de la servucción”. [En línea]. Disponible en: www.sobrepapel.com [Accesado el 14 de marzo de 2008].

Salvi, Fabiana (2014). Nuevo comportamiento del consumidor: la influencia del e-wom (electronic word-of-mouth) en relación a la lealtad de los clientes en el sector hotelero. Tesis doctoral. Universitat de les Illes baleares. https://www.tesisenred.net/bitstream/handle/10803/364766/tfs 1de1.pdf?sequence=1 \&isAllowed $=\mathrm{y}$ 
Sánchez, I. R. y Figueroa, V. M. (2008). “Marxismo y pensamiento neoclásico en torno al enfoque social de la inversión". En Colectivo de autores (2004). La economía política marxista. Reflexiones para un debate. Editorial Félix Varela. La Habana.

Sánchez, R. (2010). "La economía política y la teoría económica: desafíos para Cuba”. En Colectivo de autores (2004). La economía política marxista. Reflexiones para un debate. Editorial Félix Varela. La Habana.

Sandhusen L. Richard, Del libro: «Mercadotecnia», Sexta edición, de Compañía Editorial Continental, 2012, Pág. 385.

Sanisaca, C. E. (2012). Manual operativo de procesos para hoteles. Tesis para la obtención del título de ingeniero en turismo. Universidad de Cuenca, Ecuador.

Saris, W. E. y Gallhofer, I. (2007). "Estimation of the effects of measurement characteristics on the quality of survey questions". Survey Research Methods. Volumen 1. Número 1.

Serrano, A.; López, M.; Gómez, R. (2007). Gestión de la calidad y turismo: revisión e implicaciones para futuras investigaciones. Cuadernos de turismo, No. 20, pp. 251266. Universidad de Murcia. Recuperado el 15/01/2013, de: http://www.redalyc.org/ pdf/398/39802011.pdf

Sharma, A. y Mehta, V. (2004). "Service quality perceptions in financial services: a case study of banking services”. Journal of Services Research. Volumen 4. Número 2.

Spencer-Matthews, S y Lawley, M. (2006). The role of improving customer service: Isuess in customer contact managment. European Journal of Marketing. Vol 40: num. $1 / 2$.

Stanton, W. J., Etzel, M. J. y Walter, B. J. (2004). Fundamentos de Marketing. $13^{\mathrm{a}}$ Ed. México, D.F: McGraw Hill. 
Talaya, A. E. y Lara, E. R. (2008). Análisis del comportamiento de la demanda hotelera y su posible interferencia por el sistema de clasificación de la oferta. http://www.ucm.es/BUCM/cee/doc/0024/03010024.htm

Thompson Iván, «Mercadotecnia de servicios», cuarta Edición, de Editorial Innovación, 2006, Pág. 385.

Tsaur, S. H. y Lin, Y. C. (2004). "Promoting service quality in tourist hotel: the role of HRM practices and service behavior”. Tourism Management. Número 5.

Veloz, C. y Vasco, J. (2016). Calidad en el servicio de las empresas hoteleras de segunda categoría. Revista Ciencia UNEMI. Vol. 9, No. 18, Junio 2016, pp. 19 - 25.

Vijayadurai, J. (2008). Service Quality, Customer Satisfaction and Behavioral Intention in Hotel Industry. Journal of Marketing and Communication, (3)3, 14-26 